OPEN ACCESS

Edited by:

Meixue Zhou,

University of Tasmania, Australia

Reviewed by:

Jianfeng Weng,

Chinese Academy of Agricultural

Sciences (CAAS), China

Yubi Huang,

Sichuan Agricultural University, China

*Correspondence:

Zefeng Yang

zfyang@yzu.edu.cn

Chenwu Xu

qt/s@yzu.edu.cn

tThese authors have contributed equally to this work.

Specialty section: This article was submitted to

Plant Abiotic Stress,

a section of the journal

Frontiers in Plant Science

Received: 06 January 2017

Accepted: 17 February 2017

Published: 01 March 2017

Citation:

Li P, Cao W, Fang $H, X u S$, Yin S,

Zhang $Y$, Lin D, Wang J, Chen $Y$,

$X U$ C and Yang Z (2017)

Transcriptomic Profiling of the Maize

(Zea mays L.) Leaf Response

to Abiotic Stresses at the Seedling

Stage. Front. Plant Sci. 8:290.

doi: 10.3389/fpls.2017.00290

\section{Transcriptomic Profiling of the Maize (Zea mays L.) Leaf Response to Abiotic Stresses at the Seedling Stage}

\author{
Pengcheng Lit, Wei Caot, Huimin Fang, Shuhui Xu, Shuangyi Yin, Yingying Zhang, \\ Dezhou Lin, Jianan Wang, Yufei Chen, Chenwu Xu* and Zefeng Yang* \\ Jiangsu Provincial Key Laboratory of Crop Genetics and Physiology/Co-Innovation Center for Modern Production \\ Technology of Grain Crops, Key Laboratory of Plant Functional Genomics of Ministry of Education, Yangzhou University, \\ Yangzhou, China
}

Abiotic stresses, including drought, salinity, heat, and cold, negatively affect maize (Zea mays L.) development and productivity. To elucidate the molecular mechanisms of resistance to abiotic stresses in maize, RNA-seq was used for global transcriptome profiling of B73 seedling leaves exposed to drought, salinity, heat, and cold stress. A total of 5,330 differentially expressed genes (DEGs) were detected in differential comparisons between the control and each stressed sample, with 1,661, 2,019, 2,346, and 1,841 DEGs being identified in comparisons of the control with salinity, drought, heat, and cold stress, respectively. Functional annotations of DEGs suggested that the stress response was mediated by pathways involving hormone metabolism and signaling, transcription factors (TFs), very-long-chain fatty acid biosynthesis and lipid signaling, among others. Of the obtained DEGs (5,330), 167 genes are common to these four abiotic stresses, including 10 up-regulated TFs (five ERFs, two NACs, one ARF, one MYB, and one HDZIP) and two down-regulated TFs (one b-ZIP and one MYB-related), which suggested that common mechanisms may be initiated in response to different abiotic stresses in maize. This study contributes to a better understanding of the molecular mechanisms of maize leaf responses to abiotic stresses and could be useful for developing maize cultivars resistant to abiotic stresses.

Keywords: Zea mays L., abiotic stress, RNA-Seq, differentially expressed genes (DEGs), expression pattern

\section{INTRODUCTION}

Maize (Zea mays L.), a widely grown staple food, feed and industrial crop, plays a critical role in supporting the growing world population. The production process of maize is highly dependent on suitable environmental factors (Gong et al., 2014). However, reduction in the availability and quality of arable land and water resources as well as frequent extreme weather can cause many different types of abiotic stresses, such as salinity, drought, and extreme temperatures (heat, cold, and freezing) (Krasensky and Jonak, 2012). These major abiotic environmental stressors seriously affect crop development and constrain agronomical yield worldwide. Abiotic stresses may be responsible for a yield reduction of over 50\% in major crop plants globally (Mahajan and Tuteja, 2005; Funk and Brown, 2009; Rodziewicz et al., 2014; de Zelicourt et al., 2016). In China, 60\% of 
the maize plants are located in arid areas, and a $20-30 \%$ yield loss per year occurs in maize due to drought (Gong et al., 2014). The seedling stage of maize is especially sensitive to abiotic stresses; seedling damage can lead to stunted development and death (Peleg and Blumwald, 2011), resulting in reduced production or even rejection and incurring significant economic costs. Thus, seedling damage from abiotic stress is a subject of great concern in maize production.

Abiotic stresses can induce physiological, molecular and biochemical changes that disturb various cellular and whole-plant processes, which in turn negatively influence the development and yield of crops. Cell membranes may become disorganized, osmotic stress could be altered, proteins may lose activity or be denatured, and high levels of reactive oxygen species (ROS) could result in oxidative damage (de Zelicourt et al., 2016). These cellular changes can result in damaged cell membrane integrity, restrained photosynthesis, and dysfunctional metabolism, all of which subsequently disturb growth and development, reduce fertility, and induce premature senescence and even death of crops (Krasensky and Jonak, 2012). To counter these negative impacts of abiotic stresses, crops have evolved sophisticated resistance mechanisms in response to various stress factors, such as stress avoidance and stress tolerance. Stress avoidance is a protective mechanism that can help crops prevent or delay the negative impact of abiotic stresses, while stress tolerance is the acclimation of plants to stressful conditions (Krasensky and Jonak, 2012). Crop responses to various abiotic stresses occur at all levels of organization, including cellular responses, metabolic changes, and transcriptional regulation of gene expression. At the cellular level, crops can adjust membrane systems and modify the cell wall architecture. Several compatible solutes (e.g., proline, raffinose) can be produced to help stabilize proteins and cellular structures (Valliyodan and Nguyen, 2006; Munns and Tester, 2008). One of the fastest metabolic responses of crops is the biosynthesis of abscisic acid (ABA), which can regulate stomatal closure to reduce water loss to maintain cellular growth (Peleg and Blumwald, 2011). All of these responses are controlled at the molecular level by regulating the expression of genes involved in the synthesis of osmoprotectants and transporters and of genes encoding regulatory proteins such as protein kinases, phosphatases, and transcription factors (TFs; Sakuma et al., 2006; Krasensky and Jonak, 2012). Significant progress has been made in understanding the molecular mechanisms of plant responses to abiotic stress factors. ABA is called a "stress hormone," the discovery of ABA synthesis, perception, signaling, and transportation is a breakthroughs to understand the essential for the ability of plants to adapt to abiotic stresses (Peleg and Blumwald, 2011; Qin et al., 2011). The functions of TFs in stress tolerance have received much attention, many TFs belonging to AP2/EREBP, MYB, WRKY, NAC, bZIP families have been found to be involved in various abiotic stresses and some TF genes have also been engineered to improve stress tolerance in model and crop plants (Qin et al., 2011; Wang H.Y. et al., 2016). In addition, hormone cross-talk and lipid signaling also play a vital role in response to abiotic stresses (Peleg and Blumwald, 2011; Qin et al., 2011; Hou et al., 2016). Improvement of resistance to abiotic stresses is considered to be the most cost-effective management approach to prevent or reduce the hazards of abiotic stresses in maize (Mahajan and Tuteja, 2005). However, the molecular mechanisms of resistance to various abiotic stresses in maize are not well understood.

Abiotic stresses, especially salinity, drought, heat, and cold stress, are becoming the major threat to yield in the primary maize production regions. Utilization of maize cultivars with desirable resistance to salinity, drought, heat, or cold stress is the most cost-effective approach for preventing stress damage. Resistance to salinity, drought, heat, and cold stress in maize is a very complex abiotic stress-responsive mechanism. Investigations of the molecular mechanisms of maize response to salinity, drought, heat, or cold stress are needed to facilitate the development of elite resistant maize varieties and more effective management strategies. RNA-seq is a powerful technology for whole genome gene expression profile analysis and is especially useful for studying complex gene regulatory networks (McGettigan, 2013). To gain a comprehensive understanding of the molecular mechanisms involved in the response to salinity, drought, heat, or cold stress in the seedling stage of maize, RNA-seq was used to obtain the transcriptomic profiles of B73 seedling leaves in response to salinity, drought, heat, or cold stress at the whole-genome level. Differentially expressed genes (DEGs) were identified by comparisons between the control and the abiotic stress samples, and these DEGs were compared between the different stress samples to detect the unique and common genes and pathways responding to different abiotic stresses in maize. This study advances the understanding of the molecular responses to abiotic stresses in maize, which could lead to improved strategies for the development of new resistant maize cultivars.

\section{MATERIALS AND METHODS}

\section{Plant Material and Treatments}

B73 maize seedling plants were grown in plant growth chambers with controlled conditions: $28 / 22^{\circ} \mathrm{C}$ during a $14 / 10 \mathrm{~h}$ light/dark cycle, light density of $250-300 \mathrm{mmol} \mathrm{m}^{-2} \mathrm{~s}^{-1}$. Each black plastic pot $(10.0 \mathrm{~cm} \times 10.0 \mathrm{~cm})$ was filled with soil and watered every 2 days. When the third leaves were fully expanded, the plants were subjected to salinity, drought, heat or cold. For high salt stress, plants were watered with $200 \mathrm{mM} \mathrm{NaCl}$ for $2 \mathrm{~h}$ prior to tissue collection. For drought treatment, the 6-day-old maize seedlings were grown without watering until their third leaves were fully expanded. For heat and cold stress treatments, seedlings were incubated at $42^{\circ} \mathrm{C}$ and $4^{\circ} \mathrm{C}$ for $2 \mathrm{~h}$, respectively. After treatments, the third leaves were collected and immediately stored in liquid nitrogen for further analysis. Two independent experimental replicates were performed.

\section{RNA Isolation and cDNA Library Construction}

The leaves sampled from control and stress treatments were collected for RNA isolation and cDNA library construction. Two replicates were prepared for each sample, resulting in 10 libraries 
that were used for transcriptome sequencing using the Illumina HiSeq X Ten system.

Total RNA of maize seedling leaves was isolated using an RNeasy ${ }^{\circledR}$ Plant Mini kit (Qiagen, Shanghai, China), according to the manufacturer's protocol. All RNA samples were treated with RNase-free DNase I. A NanoDrop ${ }^{\circledR} 2000$ spectrophotometer (Thermo Scientific, Wilmington, DE, USA), a Qubit ${ }^{\circledR}$ Fluorometer 2.0 (Life Technologies, Carlsbad, CA, USA) and an Agilent 2100 bioanalyzer (Agilent Technologies, Santa Clara, CA, USA) were used to test the concentration and integrity of RNA samples, and confirm that all RNA samples had an integrity value $>6.5$.

\section{Mapping of Sequencing Reads and Quantification of Gene Expression}

The clean data were obtained by removing adapters, low-quality reads, and reads containing poly- $\mathrm{N}$ from the raw data. The Q20 and Q30 values, GC content, and sequence duplication levels were calculated for the clean data. The clean data were used for further analysis. The sequencing data were deposited in the NCBI Short Read Archive database with the accession number SRP080208.

High quality reads were aligned to the $\mathrm{B} 73$ reference sequence (AGPv3 release $31^{1}$ ) using TopHat (v2.0.9). HTSeq (v0.5.3) was used to count the read numbers mapped to each gene (Robinson and Oshlack, 2010; Anders et al., 2015). The FPKM (Fragments Per Kilobase of exon model per Million mapped reads) of each gene was calculated based on the length of the gene and read count mapped to it (Mortazavi et al., 2008).

\section{Expression Analysis and Enrichment Analysis of Differentially Expressed Genes}

The DEGs were detected with the Bioconductor package 'edgeR' in $\mathrm{R}$ between control and stress samples. The resulting $p$-values were adjusted using Benjamini and Hochberg's approach to control the false discovery rate (Benjamini and Hochberg, $1995)$. Genes with an adjusted $p$-value $(q$-value $) \leq 0.05$ and an absolute value of $\log _{2}$ fold changes $(\mathrm{FC}) \geq 1$ were considered as differentially expressed.

Gene Ontology (GO) and Kyoto Encyclopedia of Genes and Genomes (KEGG) analyses were performed to identify which DEGs were significantly enriched in GO terms or metabolic pathways. GO enrichment analysis of the DEGs was conducted using GOseq $\mathrm{R}$ packages based on the Wallenius non-central hyper-geometric distribution (Young et al., 2010), which can adjust for gene length bias in DEGs. GO terms with corrected $p$-value $(q$-value $) \leq 0.05$ were considered significantly enriched among the DEGs. KOBAS (v2.0.12) software was used to enrich the DEGs in the KEGG pathways (Mao et al., 2005). A corrected $p$-value $(q$-value $) \leq 0.05$ was the threshold for significantly enriched KEGG pathways. The web-based system Plant MetGenMAP was used to assign DEGs to metabolic pathways (Joung et al., 2009). The Plant Transcription Factor

${ }^{1} \mathrm{ftp}: / / \mathrm{ftp}$.ensemblgenomes.org/pub/plants/release-31/gff3/zea_mays
Database v3.0 was used to assign DEGs to different TF families ${ }^{2}$.

\section{qRT-PCR Analysis}

To validate the repeatability of gene expression obtained by RNA-seq, eight DEGs were randomly selected for validation by qRT-PCR. Independent RNA with two replicates of the maize seedling leaves from control and four abiotic stresses was prepared for qRT-PCR analysis. RNA extraction and quality control were performed as described above. Gene specific primers (Supplementary Table S1) were designed according to the sequences of the eight genes using QuantPrime ${ }^{3}$. The relative expression levels of the genes were calculated using the $2^{-\Delta \Delta \mathrm{Ct}}$ method (Pfaffl, 2001; Schmittgen and Livak, 2008), which represents the $\mathrm{C}_{\mathrm{T}}$ (cycle threshold) difference between the reference Actin gene and the target gene product.

\section{RESULTS}

\section{RNA-Seq and Transcriptome Profiles of Maize Leaves in Response to Abiotic Stresses}

Seedling plants of the maize inbred line B73 were either subjected to salinity, drought, heat, and cold stress conditions or grown in normal conditions (control). The total RNA of leaves from these seedling plants was sequenced using an Illumina system. We performed transcriptomic analysis of the five samples (Control, Salinity, Drought, Heat, and Cold), with two biological replicates for each condition, to profile the maize response to abiotic stresses. The RNA-seq analysis yielded 46.0-68.0 million raw reads per biological replicate with an average read length of $150 \mathrm{bp}$ (Table 1). After filtering out adapter and low-quality sequences, approximately $74.4 \mathrm{~Gb}$ of clean bases were obtained in the 10 transcriptome libraries. Of the clean reads, 77.54$82.97 \%$ were uniquely mapped to the maize reference genome sequence. The normalized FPKM was used to quantify the gene expression level (Trapnell et al., 2010). Pearson correlation analysis (Supplementary Table S2) and hierarchical cluster analysis (Figure 1A) revealed that the gene expression data of the five samples were highly reproducible between the two biological replicates of each sample, and the transcriptomes of maize seedling leaves under drought and salinity stress clustered in close proximity to each other, while the maize transcriptome under heat stress formed a separated cluster.

A gene was considered to be active when more than five reads were uniquely mapped on the maize reference genome sequence under at least one condition. In total, 27,235 of 39,625 genes were active (Figure 1B). Among the active genes $(27,235)$, 24,208 were expressed during salinity stress, 23,983 during drought stress, 22,992 during heat stress, and 22,794 during cold stress, while 23,865 were expressed in the control condition. A total of 20,101 genes were expressed in all five samples, while 1,111 genes were specifically expressed in only one sample. The

\footnotetext{
${ }^{2}$ http://planttfdb.cbi.pku.edu.cn/

${ }^{3} \mathrm{http} / /$ quantprime.mpimp-golm.mpg.de/
} 
TABLE 1 | Summary of the sequence data from Illumina sequencing.

\begin{tabular}{|c|c|c|c|c|c|c|}
\hline Sample & Replicate & Raw reads & Clean reads & Mapped reads & Mapped unique reads & Mapping ratio \\
\hline Control & 1 & $54,477,760$ & $49,675,389$ & $44,635,503$ & $43,244,605$ & $89.85 \%$ \\
\hline Control & 2 & $68,407,916$ & $62,928,805$ & $56,727,053$ & $54,894,173$ & $90.14 \%$ \\
\hline Salinity & 1 & $58,631,264$ & $54,058,240$ & $50,067,776$ & $48,648,028$ & $92.62 \%$ \\
\hline Salinity & 2 & $56,479,100$ & $51,697,101$ & $46,452,250$ & $45,107,142$ & $89.85 \%$ \\
\hline Drought & 1 & $50,139,498$ & $45,461,544$ & $40,658,089$ & $39,532,523$ & $89.43 \%$ \\
\hline Drought & 2 & $48,237,130$ & $43,045,649$ & $38,406,511$ & $37,403,429$ & $89.22 \%$ \\
\hline Heat & 1 & $52,812,100$ & $47,787,827$ & $43,793,437$ & $42,479,949$ & $91.64 \%$ \\
\hline Heat & 2 & $53,198,796$ & $48,311,290$ & $43,055,090$ & $41,908,330$ & $89.12 \%$ \\
\hline Cold & 1 & $55,216,014$ & $50,007,363$ & $44,881,791$ & $43,377,179$ & $89.75 \%$ \\
\hline Cold & 2 & $46,954,284$ & $42,754,619$ & $37,962,983$ & $36,756,281$ & $88.79 \%$ \\
\hline
\end{tabular}

Mapping ratio $=$ Mapped reads $/$ All reads.
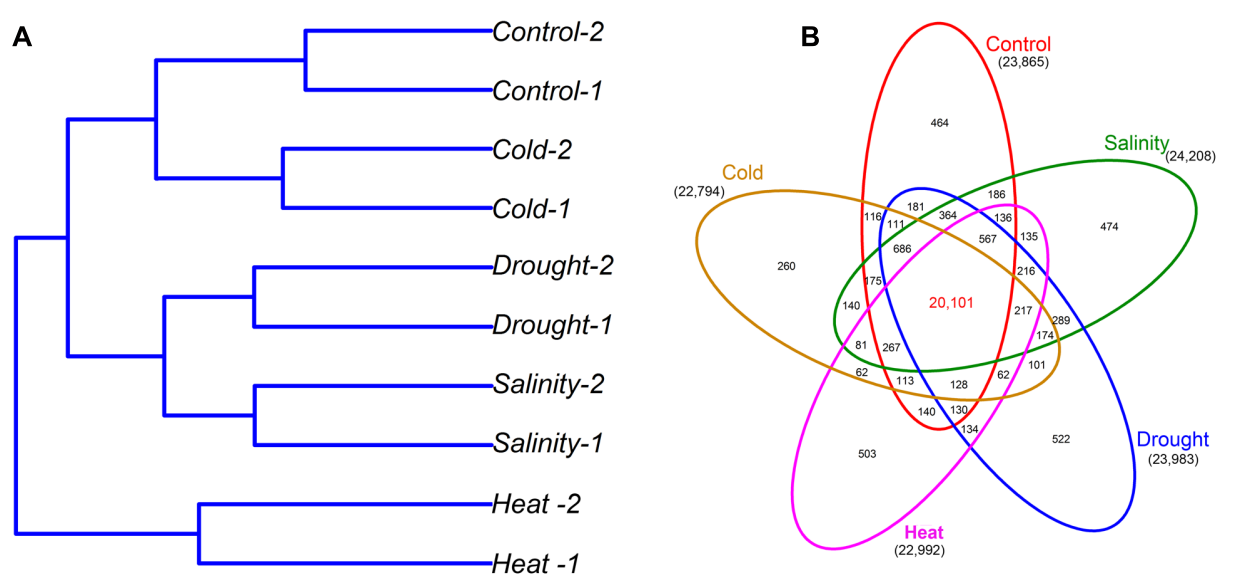

FIGURE 1 | Hierarchical clustering of the 10 RNA-seq samples based on Euclidian distance (A), and overlap of active genes under different stress conditions (B).

Control, Salinity, Drought, Heat, and Cold samples contained $464,474,522,503$, and 260 specifically expressed genes, respectively. Among the drought stress-specific genes, six GO terms (GO:0009607, response to biotic stimulus; GO:0010876, lipid localization; GO:0006869, lipid transport; GO:0030243, cellulose metabolic process; GO:0030244, cellulose biosynthetic process; GO:0005976, polysaccharide metabolic process) were significantly enriched, and the salinity stress-specific genes were significantly enriched in four GO terms (GO:0016684; oxidoreductase activity; GO:0004601, peroxidase activity; GO:0016209, antioxidant activity; GO:0030528, transcription regulator activity). However, the specifically expressed genes of heat and cold stresses did not show enrichment in any GO term.

\section{Identification and Analysis of Stress-Specific Differentially Expressed Genes (DEGs)}

Each stress sample was compared with the control to identify DEGs ( $q$-value $\leq 0.05$ and $\left|\log _{2} \mathrm{FC}\right| \geq 1$ ), and 5,330 DEGs were obtained from these four comparisons (Salinity vs. Control, Drought vs. Control, Heat vs. Control, and Cold vs. Control). From these pairwise comparisons with the control sample, we identified 1,661 (971 up- and 690 down-regulated) DEGs in the salinity stress sample, 2,019 (982 up- and 1,037 down-regulated) in the drought stress sample, 2,346 (1481 up- and 865 downregulated) in the heat stress sample and 1,841 (888 up- and 953 down-regulated) in the cold stress sample. Under salinity and heat stress, the number of up-regulated DEGs was much higher than the number of down-regulated genes (Figure 2A).

By comparing the four stress responses at the gene level, 167 DEGs were identified that were common to salinity, drought, heat, and cold stress, including 57 up- and 110 down-regulated genes (Figure 2B). We also identified hundreds of genes specific for salinity (365 up- and 166 down-regulated), drought (450 upand 436 down-regulated), heat (957 up- and 436 down-regulated) and cold stress (529 up- and 506 down-regulated).

\section{Functional Classification of DEGs}

A GO analysis was performed to determine the function of the identified DEGs. The 5,330 DEGs were enriched in $51 \mathrm{GO}$ terms in the molecular function and biological process categories (Supplementary Figure S1). Four GO terms included more than 500 DEGs. Catalytic activity (GO:0003824) and transferase activity (GO:0016740), with 1,661 and 588 DEGs, respectively, 


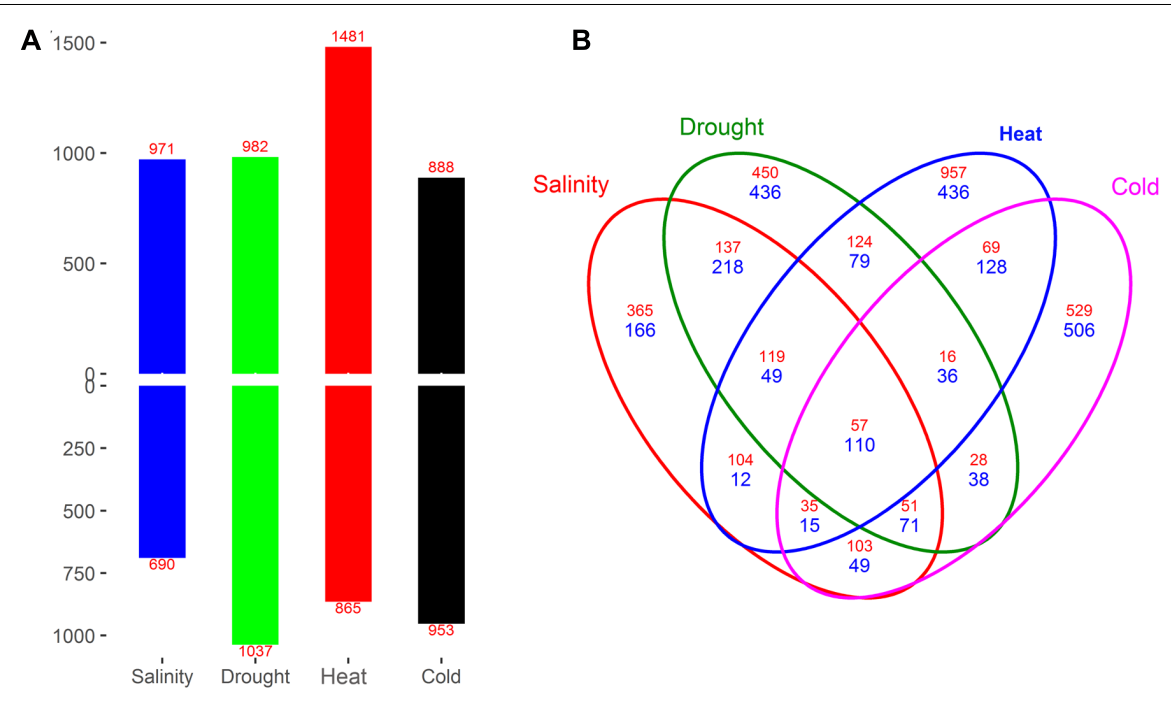

FIGURE 2 | Numbers of differentially expressed genes (DEGs) in the four pairwise comparisons of the control and stress treatments (A), and overlap between DEGs (B). DEGs from Salinity vs. Control, Drought vs. Control, Heat vs. Control, and Cold vs. Control comparisons are indicated by Salinity, Drought, Heat, and Cold, respectively. The number of up-regulated and down-regulated genes is indicated by red and blue font, respectively.

were the two most dominant terms in the molecular function category, while metabolic process (GO:0008152) and biological regulation (GO:0065007), with 1,916 and 653 DEGs, respectively, were the first two major terms in the biological process category.

Among the DEGs identified between the stress and control samples, 70, 84, 46, and 19 GO terms were enriched in the comparison of salinity, drought, heat, and cold stress versus control, respectively. To determine the transcriptomic changes that occur in response to various abiotic stresses, the enriched GO terms under different stress conditions were compared, and all commonly enriched GOs are summarized in Figure 3. Four GO terms (GO:0009737, response to ABA; GO:0009414, response to water deprivation; GO:0009409, response to cold and GO:0006950, response to stress) were enriched in all datasets, and all four were related to stimulus responses. In addition, 10 and 34 GO terms were commonly enriched in maize leaves under three and two types of abiotic stresses, respectively. There were 105 GO terms uniquely enriched in one comparison (Salinity vs. Control, Drought vs. Control, Heat vs. Control, or Cold vs. Control).

To further investigate the biological functions of these DEGs, pathway-based analysis was conducted using KEGG. We identified 11 pathways that were significantly enriched in comparisons of stress samples versus the control (Salinity vs. Control, Drought vs. Control, Heat vs. Control, or Cold vs. Control), including two pathways enriched in two comparisons and nine stress-specific pathways (Supplementary Figure S2). It is worth noting that the "Protein processing in endoplasmic reticulum" pathway was enriched in the Drought vs. Control comparison, and several genes in the "Protein processing in endoplasmic reticulum" pathway were differentially expressed in maize seedling leaves under salinity, heat, and/or cold stresses. Moreover, the "Carbon metabolism," "starch and sucrose metabolism," and "carbon fixation" pathways were significantly enriched in maize seedling leaves in response to heat stress (Supplementary Figure S2).

\section{Dynamic Expression of Transcription Factors in Response to Abiotic Stresses}

Next, the DEGs encoding TFs were analyzed. A total of 403 DEGs encoding TFs were identified in maize seedling leaves in response to salinity, drought, heat, and cold abiotic stresses, and these TFs belonged to 43 TF families. Most of the identified DEGs encoded members of the ERF, MYB, bZIP, bHLH, WRKY, NAC and MYBrelated TF families (Figure $\mathbf{4 A}$ ), and $14 \mathrm{TF}$ families included more than 9 differentially expressed TFs. The ERF family, with 58 DEGs, was the largest TF family responding to abiotic stresses, including 26,18,17, and 33 DEGs in maize seedling leaves under salinity, drought, heat, and cold stress, respectively. A total of 38 DEGs belonging to the MYB family were identified, including 18 , 22,14 , and 8 DEGs in maize leaves under salinity, drought, heat, and cold stress conditions, respectively. Most of the differentially expressed ERFs and MYBs were up-regulated; conversely, G2-like and $A R F$ s were typically down-regulated (Figure 4B). The TFs had different expression patterns in maize seedling leaf responses to salinity, drought, heat and cold stresses, which suggested that maize possesses a wide variety of abiotic stress resistance mechanisms.

\section{Expression of Hormone Biosynthesis and Signal Transduction Genes in Response to Different Abiotic Stresses}

Approximately, 50 DEGs involved in hormone biosynthesis and signal transduction pathways, such as the ABA, jasmonic acid (JA), ethylene (ET), and auxin (IAA) pathways, were identified. Moreover, 21 DEGs encoding ABA biosynthesis and catabolism enzymes and ABA receptors were obtained in 


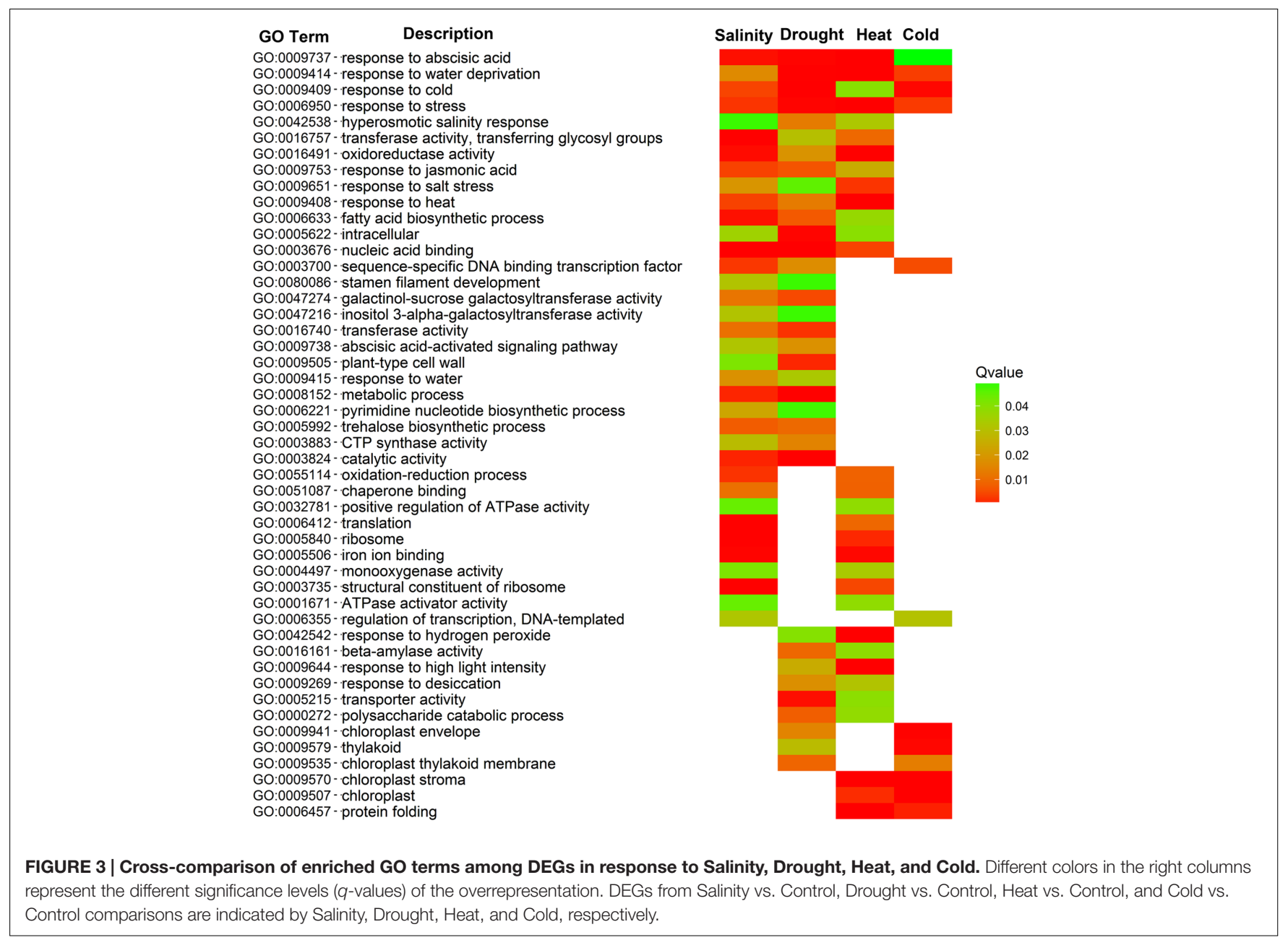

comparisons between stress samples and the control (Figure 5). As expected, the expression levels of most ABA biosynthesis enzyme genes were up-regulated in maize seedling leaves under salinity, drought, heat, and cold stresses, including $\mathrm{BCH}$ ( $\beta$-carotene hydroxylase) and NECD (9-cis-epoxycarotenoid dioxygenase). Two up-regulated $\mathrm{BCH}$ s (GRMZM2G382534 and GRMZM2G152135) were identified, with GRMZM2G382534 showing up-regulation under salinity, drought and heat stress conditions. The expression levels of $A B A 1, V E D$ (violaxanthin de-epoxidase) and $A B A 4$ were not significantly affected by salinity, drought, heat, or cold stress. Two up-regulated NECDs were identified, including $v p 14$ (GRMZM2G014392), the first cloned $N E C D$ gene in maize, which was significantly induced 10.7- to 60.8 -fold in all four stress samples. The transcriptomic levels of $A A O$ s slightly increased in maize seedling leaves in response to different treatments. CYP707As, encoding ABA catabolism enzymes, showed different expression patterns in the four treatment samples. However, the expression levels of three DEGs encoding ABA effectors were determined in response to salinity, drought, heat, and cold stresses. Four PYR1/PYLs displayed obviously decreased expression levels in drought stress, while only one PYR1/PYL was significantly down-regulated in cold stress, and none of the PYR1/PYLs were affected by salinity or heat treatment. One PP2C and three SnRKs of maize seedling leaves were up-regulated under drought or heat stress. We also detected 17, 5, and 7 DEGs involved in the JA, ET, and IAA biosynthesis pathways, respectively. Most of these phytohormone biosynthesis-related genes were up-regulated in maize seedling leaves in response to abiotic stresses, but their expression levels clearly varied by treatment condition (Figure 6 and Supplementary Dataset S2). These results indicated that phytohormone biosynthesis was reprogrammed under different abiotic stress conditions.

\section{Very-Long-Chain Fatty Acid and Lipid Signaling in Response to Abiotic Stresses}

Cuticular waxes, consisting mostly of very-long-chain fatty acids (VLCFAs) and their derivatives, play crucial roles in protecting plants against abiotic stresses (Wang et al., 2015; Wang M.L. et al., 2016). In this study, 13 DEGs involved in VLCFA and wax ester biosynthesis pathways were identified (Figure 7), including 10 genes that were up-regulated and 3 that were down-regulated in response to salinity, drought, heat and cold stresses. The expression levels of these 10 up-regulated DEGs increased 3.0- 

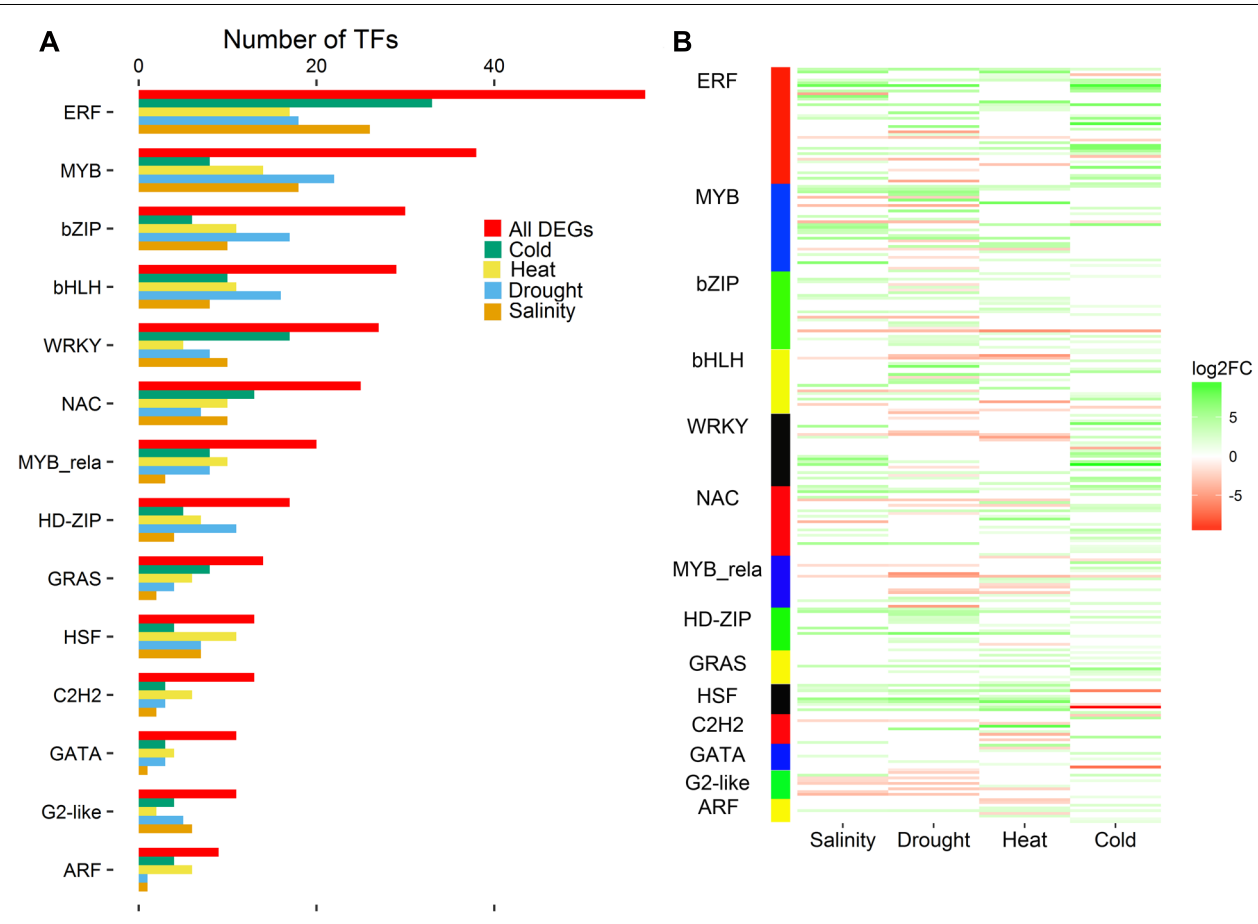

FIGURE 4 | Distribution of DEGs into 14 major transcription factor families that include more than nine DEGs (A). Heatmap of differentially expressed transcription factors in the four pairwise comparisons of control and stress treatments (B). Expression values of genes are presented as FPKM-normalized $\log _{2}$-transformed counts. Green and red colors indicate up- and down-regulated transcripts, respectively. DEGs from Salinity vs. Control, Drought vs. Control, Heat vs. Control, and Cold vs. Control comparisons are indicated by Salinity, Drought, Heat, and Cold, respectively.

to 45.8 -fold in stress samples, and the expression levels of the three down-regulated DEGs decreased 3.7- to 5.2-fold in stress samples (Supplementary Dataset S2). Among the DEGs, eight genes encoding ketoacyl-CoA synthase (KCS) were identified, which were mainly up-regulated by abiotic stress. Two transcripts for fatty acyl-coA reductase (FAR) involved in the wax ester biosynthesis pathway were found in DEGs. Of these two FAR transcripts, GRMZM2G036217 was up-regulated by salinity and drought stress, while GRMZM2G480516 was down-regulated by cold stress. Phosphatidic acid, phosphoinositides, sphingolipids and other lipids are involved in the resistance to abiotic and biotic stresses in plants (Hou et al., 2016). In this study, 25 DEGs involved in sterol, sphingolipid, phospholipid, and phosphatidylcholine biosynthesis and in phospholipid/glycolipid desaturation pathways were identified in maize seedling leaves in response to salinity, drought, heat and cold stresses (Figure 7 and Supplementary Dataset S2).

\section{Validation of RNA-Seq Analysis by Quantitative Real-Time PCR (qRT-PCR)}

To validate the reliability of the gene expression data obtained by the RNA-seq analysis in maize seedling leaves, eight DEGs were randomly selected from the control and the stress samples for qRT-PCR analysis (Supplementary Table S1). The ratio of expression levels found between stress samples and the control using qRT-PCR was compared to the ratio of expression as measured by RNA-Seq. A significant correlation $\left(r^{2}=0.8747\right.$, $n=30$, Figure 8) was observed between the RNA-Seq and qRTPCR data, which confirmed the authenticity of the DEGs in this study. Thus, these comparisons of data from qRT-PCR and RNAseq analyses of B73 seedling leaves fully validated the findings from our transcriptome study.

\section{DISCUSSION}

Abiotic stresses (such as drought, salinity, heat, and cold) strongly affect crop development and yield. Worldwide, abiotic stresses cause major crop production decreases of over $50 \%$. A better understanding of the molecular mechanisms involved in the maize response to abiotic stresses would facilitate the development of stress resistant cultivars. RNA-seq is a useful approach for the identification of DEGs and regulatory mechanisms at the transcriptome level, which could provide insights into the molecular basis of the maize response to abiotic stresses (Kakumanu et al., 2012; Opitz et al., 2014; Frey et al., 2015; Zhang et al., 2015).

In this study, we selected the period of third leaves, because that it have been suggested that the seedling stage of maize is especially sensitive to abiotic stresses (Peleg and Blumwald, 2011). Three-leaf stage represents the full immature-to-mature gradient of variation in morphology, anatomy and gene expression as the leaf is undergoing functional differentiation (Li et al., 2010). An average of 23,568 active genes were identified by transcriptomic analysis in maize seedling leaves 


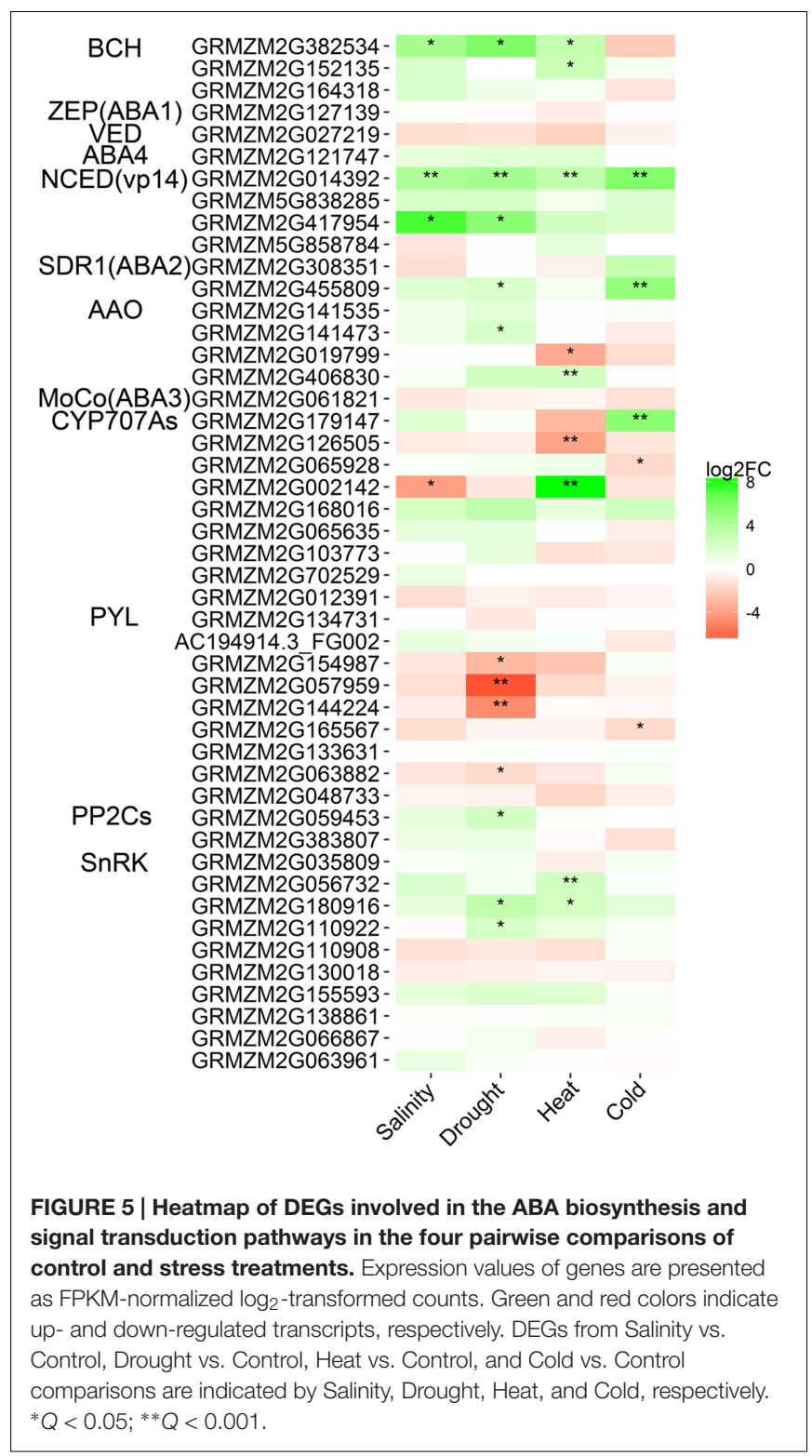

under different conditions. Interestingly, the number of active genes in each treatment sample was comparable (Figure 1). This result was similar to two previous studies (Li et al., 2010; Opitz et al., 2016), which examined 9-day-old B73 leaves and 4-day-old maize seedling roots (under water deficit conditions), respectively. However, the number of active genes obtained in our study was slightly higher than that obtained by Frey from maize leaves under heat stresses (Frey et al., 2015). In our study, 20,101 genes were expressed in all four treatments, and 260-522 genes were specifically expressed in one treatment. In total, 5,330 DEGs were identified between the control and stress samples by pairwise comparisons. The largest effect of abiotic stress on gene regulation was detected under heat stress, with 1,481 up- and 865 down-regulated DEGs identified. The number of up-regulated DEGs was much higher than the downregulated genes under salinity and heat stress, which is consistent with the results of transcriptomic profiling of European maize inbred lines (Frey et al., 2015) and Medicago falcata (Miao et al., 2015). Functional annotation of DEGs found that a number of biological pathways, including hormone metabolism and signaling, transcriptional regulation and lipid signaling, participated in the response to abiotic stress in seedling maize (Figures 3-7).

\section{DEGs Involved in ABA Biosynthesis and Signaling}

Plant hormones play critical roles in regulating the growth and development of crops under abiotic stresses (Peleg and Blumwald, 2011). One of the most studied phytohormones is ABA, which has been described as a stress hormone, and crops have been shown to adjust ABA levels in response to adverse environmental conditions (Tuteja, 2007). ABA biosynthesis and catabolism are regulated by $\mathrm{BCH}, \mathrm{ABA} 1, \mathrm{NCED}, \mathrm{ABA} 2, \mathrm{AAO}$, and CYP707A (Nambara and Marion-Poll, 2005; Peleg and Blumwald, 2011; Chan, 2012; Shan et al., 2013; Shi et al., 2014; Song et al., 2014; Miao et al., 2015; Shankar et al., 2016). Zeaxanthin is an important intermediate in the biosynthetic reaction of $\beta$-carotene to form $\mathrm{ABA} ; \beta$-carotene hydroxylases encoded by $\mathrm{BCH}$ can catalyze the biosynthesis of zeaxanthin (Finkelstein, 2013); xanthophyll cleavage by NCED is the first committed, rate-limiting step in ABA biosynthesis; and CYP707As encode enzymes that can catalyze ABA catabolism. The expression of these genes involved in ABA biosynthesis and catabolism could be activated or decreased by abiotic stresses (Tuteja, 2007; Song et al., 2014; Miao et al., 2015). In our study, the expression levels of $B C H, N E C D, A B A 2$, and $A A O$, the key genes involved in ABA biosynthesis, were significantly up-regulated in seedling maize leaves in response to salinity, drought, heat, and cold stresses. Increased expression levels of the NCED genes have been shown in both roots and leaves under abiotic stress conditions, especially in maize (Tan et al., 1997, 2003; Iuchi et al., 2001; Xiong et al., 2001). Vp14 was the first cloned NCED gene in maize (Tan et al., 1997), and its expression is significantly up-regulated by most abiotic stresses (Shan et al., 2013; Song et al., 2014; Miao et al., 2015). ABA levels in crops are the result of a delicate balance between ABA biosynthesis and catabolism (Nambara and Marion-Poll, 2005). CYP707A is an important hydroxylase involved in ABA catabolism (Finkelstein, 2013), and the CYP707A genes showed different expression patterns in response to salinity, drought, heat and cold stresses in this study (Figure 5). Different spatial and temporal expression patterns of CYP707A have suggested that CYP707As could play different physiological roles in plant development (Nambara and Marion-Poll, 2005). Our observation that genes involved in ABA biosynthesis were upregulated in response to stress indicates that the endogenous ABA level in seedling maize increases to adapt to various abiotic stresses.

Expression of components of the ABA signaling pathway, such as PYR/PYL, PP2C, and SnRK, is related to various abiotic stresses in crops (Nambara and Marion-Poll, 2005; Finkelstein, 2013; Fan et al., 2016). The expression levels of PP2Cs and 


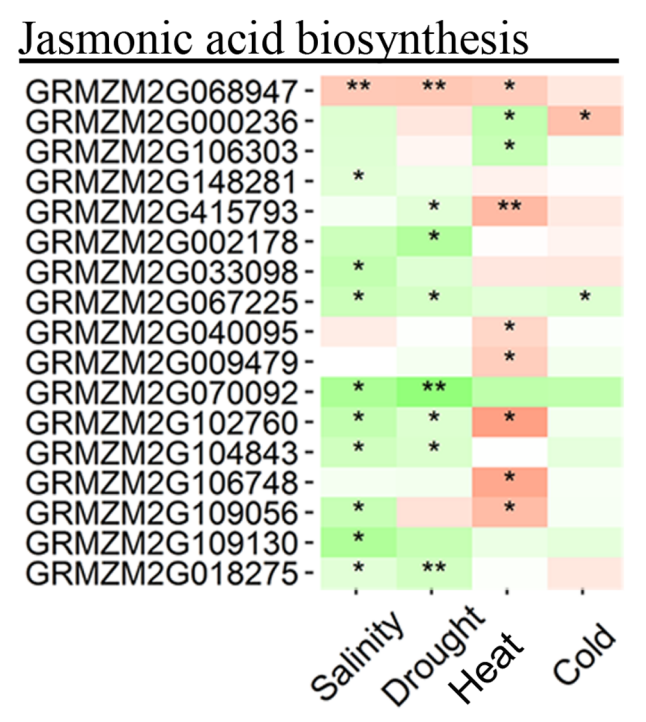

\section{Ethylene biosynthesis}

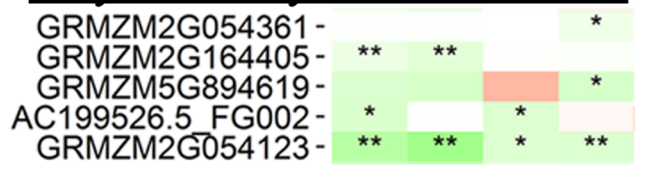

IAA biosynthesis

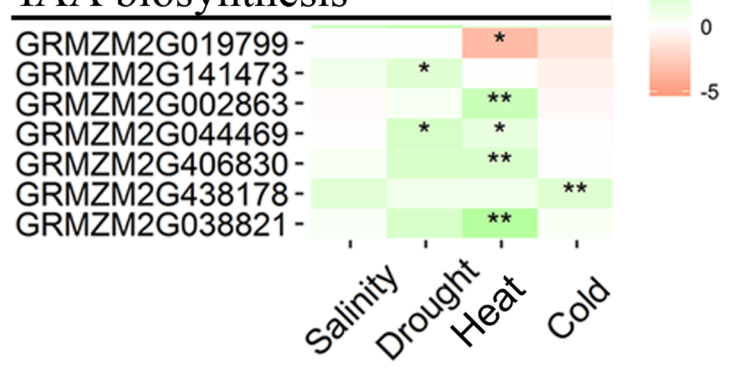

FIGURE 6 | Heatmap of DEGs involved in hormone (jasmonic acid, ethylene, and IAA) biosynthesis, photosynthesis, and carbon metabolism pathways in the four pairwise comparisons of control and stress treatments. Expression values of genes are presented as FPKM-normalized $\log _{2}$-transformed counts. Green and red colors indicate up- and down-regulated transcripts, respectively. DEGs from Salinity vs. Control, Drought vs. Control, Heat vs. Control, and Cold vs. Control comparisons are indicated by Salinity, Drought, Heat, and Cold, respectively. ${ }^{*} Q<0.05 ; * * Q<0.001$.

SnRKs were mainly up-regulated, while PYR/PYLs showed down-regulation, in maize (Fan et al., 2016) and Arabidopsis (Chan, 2012). Five PYR/PYL DEGs were identified in this study, including four in response to drought stress in seedling maize leaves and one in response to cold stress (Figure 5). In addition, only one $P P 2 C$ was identified in drought stress, and three $S n R K$ s were differentially expressed in drought and heat stress conditions. Consistent with previous reports, our transcriptomic analysis showed different expression patterns of genes involved in $\mathrm{ABA}$ biosynthesis and signaling in seedling maize in response to abiotic stresses.

\section{DEGs Encoding Transcription Factors}

Transcription factors regulate almost all aspects of plant growth and development and could orchestrate regulatory networks to improve resistance to abiotic and biotic stresses in plants (Golldack et al., 2014). Major plant TF families such as NAC, AP2/ERF, bZIP, and MYB have been documented as important regulators in plant responses to various abiotic and biotic stresses (Golldack et al., 2011; Shan et al., 2013; Shi et al., 2014; Song et al., 2014; Miao et al., 2015; Shankar et al., 2016; Wang H.Y. et al., 2016). In this study, a total of $43 \mathrm{TF}$ families containing 403 differentially expressed TFs were identified by a paired comparison. Most of the differentially expressed TFs belonged to the ERF, MYB, bZIP, bHLH, WRKY, and NAC TF families (Figure 4A). The ERF TF family was the largest class in the seedling maize leaf response to abiotic stresses, with 48 up-regulated and 10 down-regulated DEGs. A previous study identified 184 AP2/ERF genes in maize, 38 of which were involved in the response to waterlog stress (Du et al., 2014). Moreover, over-expressing three DREB1s belonging to the ERF TF family significantly improved resistance to freezing, drought, and high salinity in Arabidopsis (Gilmour et al., 1998; Jaglo-Ottosen et al., 1998; Liu et al., 1998). Numerous MYB TFs play vital roles in cell development, hormone signaling, and cuticular wax biosynthesis in crops (Seo et al., 2009, 2011). We found 18, 22, 14, and 8 differentially expressed MYBs in seedling maize leaves in response to salinity, drought, heat, and cold stresses, respectively. Notably, the over-expression of OsMYB55, a member of the MYB TF family, improved resistance to heat and drought stresses through activating the expression of resistance-related genes in maize (Casaretto et al., 2016). ABP9, which encodes a bZIP TF, could remarkably enhance the resistance to abiotic stresses such as drought, high salt, freezing temperature, and oxidative stresses in Arabidopsis (Zhang et al., 2011). In this study, 30 differentially expressed bZIP TFs were identified in response to salinity, drought, heat, and cold stresses, including eight upregulated DEGs that were found in at least two comparisons of abiotic stress samples (salinity, drought, heat, or cold stress) with the control. These results emphasized that TFs play vital roles in improving resistance to multiple abiotic stresses in maize.

\section{DEGs Involved in Very-Long-Chain Fatty Acid (VLCFA) and Lipid Signaling}

Cuticular wax, a complex mixture of hydrophobic lipids, covers the outermost surfaces of land plants and acts as a protective barrier against abiotic stresses (Bernard and Joubès, 2013; Wang M.L. et al., 2016). Cuticular wax is composed of VLCFAs (C20 to C34) and their derivatives, which are synthesized in the endoplasmic reticulum (ER; Samuels et al., 2008). Cuticular wax biosynthesis begins with the de novo $\mathrm{C} 16$ or $\mathrm{C} 18$ fatty acid synthesis, which is catalyzed by a multi-enzyme complex that includes fatty acid elongases (FAEs), $\beta$-ketoacyl-CoA synthase 


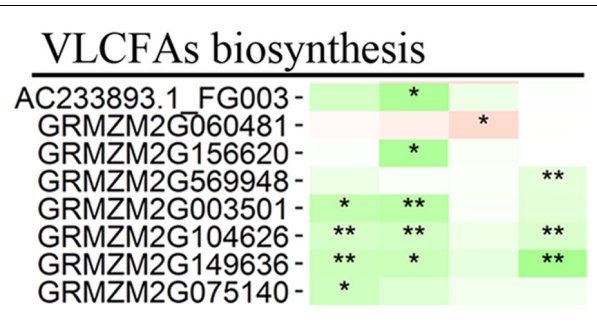

Sterol biosynthesis

\begin{tabular}{llll} 
& & \\
\hline GRMZM2G075701- & & & \\
GRMZM2G006937- & $* *$ & $*$ & \\
GRMZM2G177384- & $*$ & $*$ & \\
GRMZM2G068465- & & & $* *$ \\
GRMZM2G091456- & $*$ & & \\
GRMZM2G105787- & * & &
\end{tabular}

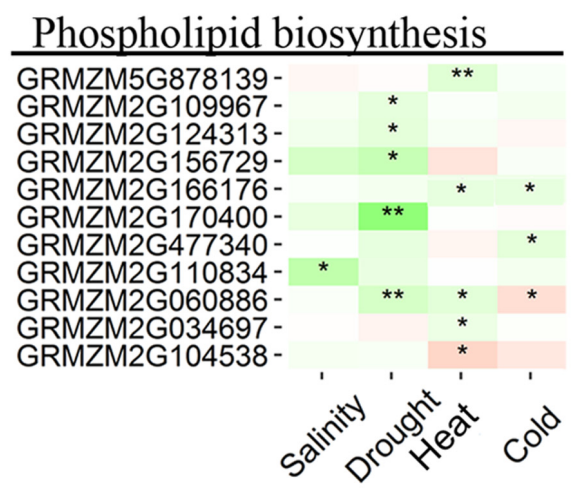

Wax esters biosynthesis

\begin{tabular}{ll|l}
\hline GRMZM2G480516- & $*$ & $*$ \\
GRMZM2G036217- & $*$ & $*$ \\
GRMZM5G872443- & $*$ & \\
GRMZM2G042356- & $* *$ \\
GRMZM2G077375- & &
\end{tabular}

Sphingolipid biosynthesis

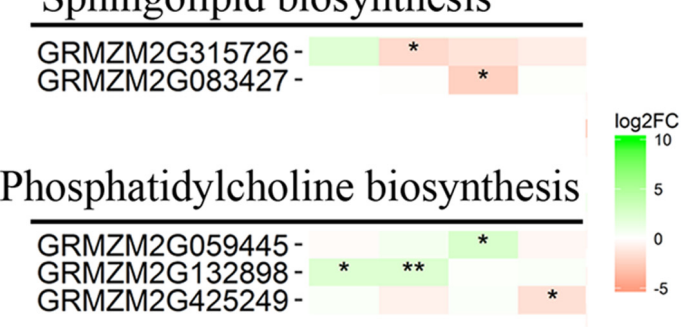

Phospholipid/ glycolipid desaturation

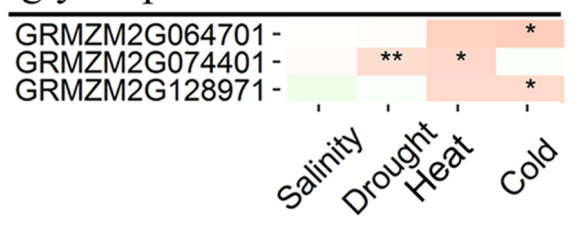

FIGURE 7 | Heatmap of DEGs involved in very-long-chain fatty acid (VLCFA) and lipid signaling in the four pairwise comparisons of control and stress treatments. Expression values of genes are presented as FPKM-normalized $\log _{2}$-transformed counts. Green and red colors indicate up-and down-regulated transcripts, respectively. DEGs from Salinity vs. Control, Drought vs. Control, Heat vs. Control, and Cold vs. Control comparisons are indicated by Salinity, Drought, Heat, and Cold, respectively. ${ }^{*} Q<0.05 ;{ }^{* *} Q<0.001$.

(KCS), $\beta$-ketoacyl-CoA reductase (KCR), and FAR, among other components (Lee and Suh, 2013). The expression of wax biosynthesis-related genes, such as FARs, KCS6, and CER1, can be highly activated by drought stress in plants (Bourdenx et al., 2011; Wang M.L. et al., 2016). In this study, eight KCS genes were significantly up-regulated in response to salinity, drought, heat, and cold stresses (Figure 7), which suggested that KCS is involved in the resistance to abiotic stresses (salinity, drought, heat, or cold stress) in maize. Additionally, two differentially expressed FAR genes were identified in response to salinity and heat stresses. As mentioned above, cuticular wax is biosynthesized in the ER (Samuels et al., 2008). In our study, the "Protein processing in endoplasmic reticulum" pathway was significantly enriched in the seedling maize response to drought; moreover, several up-regulated DEGs in the "Protein processing in endoplasmic reticulum" pathway were identified in salinity, heat and cold stress samples (Supplementary Figure S2).

Lipid, the interface between the plant cell and the environment, is an essential biomolecule for plant responses to multiple abiotic and biotic stresses (Hou et al., 2016). Steroids (Vriet et al., 2012; Senthil-Kumar et al., 2013), sphingolipids (Lynch, 2012), phospholipids (Darwish et al., 2009), phosphatidylcholines (Tasseva et al., 2004) and other lipids act as signaling molecules that can improve resistance to various stresses in plants (Hou et al., 2016). Six up-regulated DEGs involved in sterol biosynthesis were identified in response to different abiotic stresses; however, the expression levels of these DEGs involved in maize sterol biosynthesis varied based on the abiotic stress applied (Figure 7). Most of the DEGs involved in lipid molecule biosynthesis were significantly up-regulated in seedling maize in response to abiotic stresses, while 3 DEGs involved in phospholipid desaturation were down-regulated. The above analysis demonstrated that lipid regulation is a key molecular and biochemical mechanism for resistance to abiotic stresses in seedling maize.

\section{Common and Unique Molecular Mechanism Responses to Various Abiotic Stresses in Maize}

Plants often suffer from different adverse environmental conditions simultaneously (Ahuja et al., 2010), and common mechanisms have evolved to respond to various abiotic stresses. In our dataset, 167 DEGs (57 up-regulated and 110 downregulated) were identified by comparison between the control and stress sample (Figure 2). Many up-regulated DEGs, such as 


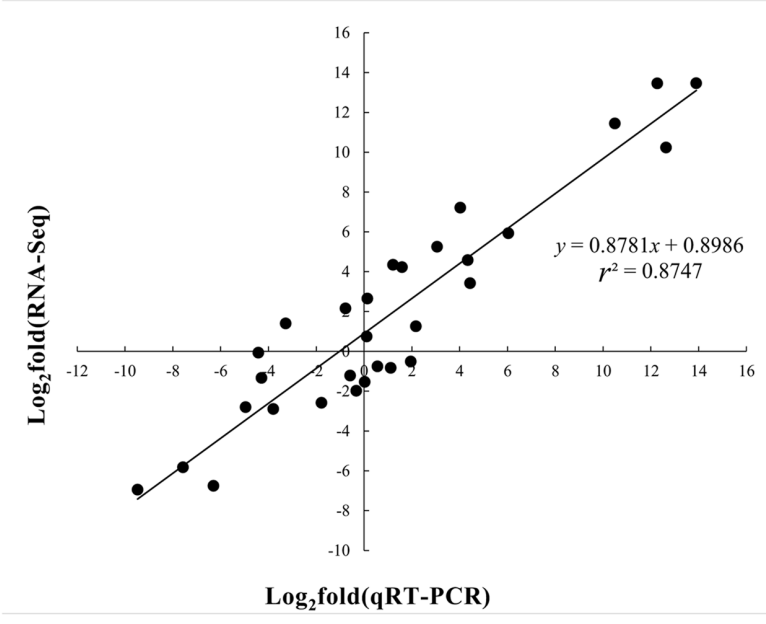

FIGURE 8 | Quantitative real-time PCR validations of DEGs characterized by RNA-seq.
GRMZM2G014392 (Vp14), GRMZM6G441368 (F-box protein), and GRMZM2G373522 (Dehydrin), were found in all four stress samples (salinity, drought, heat, and cold), while the functions of most of the down-regulated genes were unknown (Supplementary Dataset S1). Ten up-regulated TFs, belonging to five TF families (five ERFs, two NACs, one ARF, one $\mathrm{MYB}$, and one HD-ZIP), and two down-regulated TFs (one bZIP and one MYB-related) were identified in all four stress samples. These results highlighted the important role of TFs in maize resistance to various abiotic stresses. Furthermore, GO enrichment analysis found that 4, 10, and 34 GO terms were over-represented in 4, 3, and 2 stress samples, respectively. These common GO terms were primarily related to plant hormones (ABA and JA), stress response, and fatty acid biosynthetic processes (Figure 3). In agricultural production processes, various abiotic stresses typically occur simultaneously (Ahuja et al., 2010); for example, drought stress is often accompanied by salinity stress, and heat stress by drought stress. Pairwise comparisons of identified common DEGs between different stresses demonstrated that the number of DEGs in Salinity vs. Drought (355), Drought vs. Heat (203), and Heat vs. Cold (197) were the top three comparisons, only 66, 116, and 152 common DEGs in Drought vs. Cold, Salinity vs. Heat, and Salinity vs. Cold (Figure 2B). The results are consistent with hierarchical cluster analysis. Drought stress, as salt stress, causes osmotic imbalances in the plant tissues (Mahajan and Tuteja, 2005; Huang et al., 2012), plant maybe evolved similar molecular programs to adapt different stresses. This result offers a possible way to improve the multiple-stress tolerance of maize. Although several common DEGs and GO terms were identified in seedling maize in response to different abiotic stresses, most of the obtained DEGs were unique to a particular abiotic stress, which suggested that maize possesses many common and unique molecular mechanisms relating to the resistance to various abiotic stresses.

\section{CONCLUSION}

In the present study, RNA-seq was applied to detect the global transcriptional changes in seedling maize leaves in response to abiotic stresses. In total, 5,330 DEGs were identified between the control and stress samples. Genes related to hormone metabolism and signaling, TFs, VLCFA biosynthesis and lipid signaling were found to be involved in the resistance to salinity, drought, heat and/or cold stresses in seedling maize. Importantly, 167 DEGs were commonly identified in four seedling maize samples in response to salinity, drought, heat and cold stresses, which suggests that there are many common and unique molecular mechanisms related to the resistance to various abiotic stresses in maize. This study extends the understanding of the molecular mechanisms of maize leaf resistance to abiotic stresses in the seedling stage and will be useful for identifying major candidate genes and molecular markers for improving resistance to abiotic stresses in maize.

\section{AUTHOR CONTRIBUTIONS}

PL, WC, ZY, and CX conceived the experiment and made the revision of the manuscript. WC, PL, HF, SX, YZ, DL, JW, and YC performed the research and collected data. PL, WC, and SY analyzed the data and wrote the manuscript. All authors reviewed and approved this submission.

\section{ACKNOWLEDGMENTS}

This work was supported by grants from the National Key Technology Research and Development Program of MOST (2016YFD0100300), the National Natural Science Foundations (31391632, 91535103 and 31601810), the Priority Academic Program Development of Jiangsu Higher Education Institutions, the National High-tech R\&D Program (863 Program) (2014AA10A601-5), the Natural Science Foundations of Jiangsu Province (BK20150010), the Natural Science Foundation of the Jiangsu Higher Education Institutions (14KJA210005), and the Innovative Research Team of Universities in Jiangsu Province.

\section{SUPPLEMENTARY MATERIAL}

The Supplementary Material for this article can be found online at: http://journal.frontiersin.org/article/10.3389/fpls.2017.00290/ full\#supplementary-material 


\section{REFERENCES}

Ahuja, I., de Vos, R. C. H., Bones, A. M., and Hall, R. D. (2010). Plant molecular stress responses face climate change. Trends Plant Sci. 15, 664-674. doi: 10.1016/ j.tplants.2010.08.002

Anders, S., Pyl, P. T., and Huber, W. (2015). HTSeq-a Python framework to work with high-throughput sequencing data. Bioinformatics 31, 166-169. doi: 10.1093/bioinformatics/btu638

Benjamini, Y., and Hochberg, Y. (1995). Controlling the false discovery rate: a practical and powerful approach to multiple testing. J. R. Stat. Soc. B (Methodological) 57, 289-300. doi: 10.2307/2346101

Bernard, A., and Joubès, J. (2013). Arabidopsis cuticular waxes: advances in synthesis, export and regulation. Prog. Lipid Res. 52, 110-129. doi: 10.1016/j. plipres.2012.10.002

Bourdenx, B., Bernard, A., Domergue, F., Pascal, S., Léger, A., Roby, D., et al. (2011). Overexpression of Arabidopsis ECERIFERUM1 promotes wax verylong-chain alkane biosynthesis and influences plant response to biotic and abiotic stresses. Plant Physiol. 156, 29-45. doi: 10.1104/pp.111.172320

Casaretto, J. A., El-kereamy, A., Zeng, B., Stiegelmeyer, S. M., Chen, X., Bi, Y. M., et al. (2016). Expression of OsMYB55 in maize activates stress-responsive genes and enhances heat and drought tolerance. BMC Genomics 17:312. doi: 10.1186/ s12864-016-2659-5

Chan, Z. L. (2012). Expression profiling of ABA pathway transcripts indicates crosstalk between abiotic and biotic stress responses in Arabidopsis. Genomics 100, 110-115. doi: 10.1016/j.ygeno.2012.06.004

Darwish, E., Testerink, C., Khalil, M., El-Shihy, O., and Munnik, T. (2009). Phospholipid signaling responses in salt-stressed rice leaves. Plant Cell Physiol. 50, 986-997. doi: 10.1093/pcp/pcp051

de Zelicourt, A., Colcombet, J., and Hirt, H. (2016). The role of MAPK modules and ABA during abiotic stress signaling. Trends Plant Sci. 21, 677-685. doi: 10.1016/j.tplants.2016.04.004

Du, H. W., Huang, M., Zhang, Z. X., and Cheng, S. Y. (2014). Genome-wide analysis of the AP2/ERF gene family in maize waterlogging stress response. Euphytica 198, 115-126. doi: 10.1007/s10681-014-1088-2

Fan, W., Zhao, M., Li, S., Bai, X., Li, J., Meng, H., et al. (2016). Contrasting transcriptional responses of PYR1/PYL/RCAR ABA receptors to ABA or dehydration stress between maize seedling leaves and roots. BMC Plant Biol. 16:99. doi: 10.1186/s12870-016-0764-x

Finkelstein, R. (2013). Abscisic Acid synthesis and response. Arabidopsis Book 11:e0166. doi: 10.1199/tab.0166

Frey, F. P., Urbany, C., Huttel, B., Reinhardt, R., and Stich, B. (2015). Genome-wide expression profiling and phenotypic evaluation of European maize inbreds at seedling stage in response to heat stress. BMC Genomics 16:123. doi: 10.1186/ s12864-015-1282-1

Funk, C. C., and Brown, M. E. (2009). Declining global per capita agricultural production and warming oceans threaten food security. Food Sec. 1, 271-289. doi: 10.1007/s12571-009-0026-y

Gilmour, S. J., Zarka, D. G., Stockinger, E. J., Salazar, M. P., Houghton, J. M., and Thomashow, M. F. (1998). Low temperature regulation of the Arabidopsis CBF family of AP2 transcriptional activators as an early step in cold-induced COR gene expression. Plant J. 16, 433-442. doi: 10.1046/j.1365-313x.1998.00310.x

Golldack, D., Li, C., Mohan, H., and Probst, N. (2014). Tolerance to drought and salt stress in plants: unraveling the signaling networks. Front. Plant Sci. 5:151. doi: 10.3389/fpls.2014.00151

Golldack, D., Luking, I., and Yang, O. (2011). Plant tolerance to drought and salinity: stress regulating transcription factors and their functional significance in the cellular transcriptional network. Plant Cell Rep. 30, 1383-1391. doi: 10.1007/s00299-011-1068-0

Gong, F., Yang, L., Tai, F., Hu, X., and Wang, W. (2014). “Omics” of maize stress response for sustainable food production: opportunities and challenges. OMICS 18, 714-732. doi: 10.1089/omi.2014.0125

Hou, Q. C., Ufer, G. D., and Bartels, D. (2016). Lipid signalling in plant responses to abiotic stress. Plant Cell Environ. 39, 1029-1048. doi: 10.1111/pce.12666

Huang, G. T., Ma, S. L., Bai, L. P., Zhang, L., Ma, H., Jia, P., et al. (2012). Signal transduction during cold, salt, and drought stresses in plants. Mol. Biol. Rep. 39, 969-987. doi: 10.1007/s11033-011-0823-1

Iuchi, S., Kobayashi, M., Taji, T., Naramoto, M., Seki, M., Kato, T., et al. (2001). Regulation of drought tolerance by gene manipulation of 9-cis-epoxycarotenoid dioxygenase, a key enzyme in abscisic acid biosynthesis in Arabidopsis. Plant J. 27, 325-333. doi: 10.1046/j.1365-313x.2001.01096.x

Jaglo-Ottosen, K. R., Gilmour, S. J., Zarka, D. G., Schabenberger, O., and Thomashow, M. F. (1998). Arabidopsis CBF1 overexpression induces COR genes and enhances freezing tolerance. Science 280, 104-106. doi: 10.1126/ science.280.5360.104

Joung, J. G., Corbett, A. M., Fellman, S. M., Tieman, D. M., Klee, H. J., Giovannoni, J. J., et al. (2009). Plant MetGenMAP: an integrative analysis system for plant systems biology. Plant Physiol. 151, 1758-1768. doi: 10.1104/pp.109.145169

Kakumanu, A., Ambavaram, M. M. R., Klumas, C., Krishnan, A., Batlang, U., Myers, E., et al. (2012). Effects of drought on gene expression in maize reproductive and leaf meristem tissue revealed by RNA-Seq. Plant Physiol. 160, 846-867. doi: 10.1104/pp.112.200444

Krasensky, J., and Jonak, C. (2012). Drought, salt, and temperature stress-induced metabolic rearrangements and regulatory networks. J. Exp. Bot. 63, 1593. doi: $10.1093 /$ jxb/err460

Lee, S. B., and Suh, M. C. (2013). Recent advances in cuticular wax biosynthesis and its regulation in Arabidopsis. Molecular Plant 6, 246-249. doi: 10.1093/mp/ sss 159

Li, P. H., Ponnala, L., Gandotra, N., Wang, L., Si, Y. Q., Tausta, S. L., et al. (2010). The developmental dynamics of the maize leaf transcriptome. Nat. Genet. 42, 1060-U1051. doi: 10.1038/ng.703

Liu, Q., Kasuga, M., Sakuma, Y., Abe, H., Miura, S., Yamaguchi-Shinozaki, K., et al. (1998). Two transcription factors, DREB1 and DREB2, with an EREBP/AP2 DNA binding domain separate two cellular signal transduction pathways in drought-and low-temperature-responsive gene expression, respectively, in Arabidopsis. Plant Cell 10, 1391-1406. doi: 10.2307/3870648

Lynch, D. V. (2012). Evidence that sphingolipid signaling is involved in responding to low temperature. New Phytol. 194, 7-9. doi: 10.1111/j.1469-8137.2012. 04078.x

Mahajan, S., and Tuteja, N. (2005). Cold, salinity and drought stresses: an overview. Arch. Biochem. Biophys. 444, 139-158. doi: 10.1016/j.abb.2005.10.018

Mao, X. Z., Cai, T., Olyarchuk, J. G., and Wei, L. P. (2005). Automated genome annotation and pathway identification using the KEGG Orthology (KO) as a controlled vocabulary. Bioinformatics 21, 3787-3793. doi: 10.1093/ bioinformatics/bti430

McGettigan, P. A. (2013). Transcriptomics in the RNA-seq era. Curr. Opin. Chem. Biol. 17, 4-11. doi: 10.1016/j.cbpa.2012.12.008

Miao, Z. Y., Xu, W., Li, D. F., Hu, X. N., Liu, J. X., Zhang, R. X., et al. (2015). De novo transcriptome analysis of Medicago falcata reveals novel insights about the mechanisms underlying abiotic stress-responsive pathway. BMC Genomics 16:818. doi: 10.1186/s12864-015-2019-x

Mortazavi, A., Williams, B. A., McCue, K., Schaeffer, L., and Wold, B. (2008). Mapping and quantifying mammalian transcriptomes by RNA-Seq. Nat. Methods 5, 621-628. doi: 10.1038/nmeth.1226

Munns, R., and Tester, M. (2008). Mechanisms of salinity tolerance. Annu. Rev. Plant Biol. 59, 651-681. doi: 10.1146/annurev.arplant.59.032607.092911

Nambara, E., and Marion-Poll, A. (2005). Abscisic acid biosynthesis and catabolism. Annu. Rev. Plant Biol. 56, 165-185. doi: 10.1146/annurev.arplant. 56.032604 .144046

Opitz, N., Marcon, C., Paschold, A., Malik, W. A., Lithio, A., Brandt, R., et al. (2016). Extensive tissue-specific transcriptomic plasticity in maize primary roots upon water deficit. J. Exp. Bot. 67, 1095-1107. doi: 10.1093/jxb/erv453

Opitz, N., Paschold, A., Marcon, C., Malik, W. A., Lanz, C., Piepho, H. P., et al. (2014). Transcriptomic complexity in young maize primary roots in response to low water potentials. BMC Genomics 15:741. doi: 10.1186/1471-2164-15-741

Peleg, Z., and Blumwald, E. (2011). Hormone balance and abiotic stress tolerance in crop plants. Curr. Opin. Plant Biol. 14, 290-295. doi: 10.1016/j.pbi.2011.02.001

Pfaffl, M. W. (2001). A new mathematical model for relative quantification in real-time RT-PCR. Nucleic Acids Res. 29:e45. doi: 10.1093/nar/29.9.e45

Qin, F., Shinozaki, K., and Yamaguchi-Shinozaki, K. (2011). Achievements and challenges in understanding plant abiotic stress responses and tolerance. Plant Cell Physiol. 52, 1569-1582. doi: 10.1093/pcp/pcr106

Robinson, M. D., and Oshlack, A. (2010). A scaling normalization method for differential expression analysis of RNA-seq data. Genome Biol. 11:R25. doi: 10.1186/gb-2010-11-3-r25

Rodziewicz, P., Swarcewicz, B., Chmielewska, K., Wojakowska, A., and Stobiecki, M. (2014). Influence of abiotic stresses on plant proteome and 
metabolome changes. Acta Physiol. Plant. 36, 1-19. doi: 10.1007/s11738-013$1402-y$

Sakuma, Y., Maruyama, K., Qin, F., Osakabe, Y., Shinozaki, K., and Yamaguchi-Shinozaki, K. (2006). Dual function of an Arabidopsis transcription factor DREB2A in water-stress-responsive and heat-stressresponsive gene expression. Proc. Natl. Acad. Sci. U.S.A. 103, 18822-18827. doi: $10.1073 /$ pnas.0605639103

Samuels, L., Kunst, L., and Jetter, R. (2008). Sealing plant surfaces: cuticular wax formation by epidermal cells. Annu. Rev. Plant Biol. 59, 683-707. doi: 10.1146/ annurev.arplant.59.103006.093219

Schmittgen, T. D., and Livak, K. J. (2008). Analyzing real-time PCR data by the comparative CT method. Nat. Protoc. 3, 1101-1108.

Senthil-Kumar, M., Wang, K., and Mysore, K. S. (2013). AtCYP710A1 genemediated stigmasterol production plays a role in imparting temperature stress tolerance in Arabidopsis thaliana. Plant Signal. Behav. 8:e23142. doi: 10.4161/ psb. 23142

Seo, P. J., Lee, S. B., Suh, M. C., Park, M. J., Go, Y. S., and Park, C. M. (2011). The MYB96 transcription factor regulates cuticular wax biosynthesis under drought conditions in Arabidopsis. Plant Cell 23, 1138-1152. doi: 10.1105/tpc. 111.083485

Seo, P. J., Xiang, F. N., Qiao, M., Park, J. Y., Lee, Y. N., Kim, S. G., et al. (2009). The MYB96 transcription factor mediates abscisic acid signaling during drought stress response in Arabidopsis. Plant Physiol. 151, 275-289. doi: 10.1104/pp.109. 144220

Shan, X. H., Li, Y. D., Jiang, Y., Jiang, Z. L., Hao, W. Y., and Yuan, Y. P. (2013). Transcriptome profile analysis of maize seedlings in response to highsalinity, drought and cold stresses by deep sequencing. Plant Mol. Biol. Rep. 31, 1485-1491. doi: 10.1007/s11105-013-0622-z

Shankar, R., Bhattacharjee, A., and Jain, M. (2016). Transcriptome analysis in different rice cultivars provides novel insights into desiccation and salinity stress responses. Sci. Rep. 6:23719. doi: 10.1038/srep23719

Shi, G. X., Huang, F., Gong, Y., Xu, G. L., Yu, J. J., Hu, Z. B., et al. (2014). RNA-Seq analysis reveals that multiple phytohormone biosynthesis and signal transduction pathways are reprogrammed in curled-cotyledons mutant of soybean Glycine max (L.) Merr. BMC Genomics 15:510. doi: 10.1186/14712164-15-510

Song, Y. P., Ci, D., Tian, M., and Zhang, D. Q. (2014). Comparison of the physiological effects and transcriptome responses of Populus simonii under different abiotic stresses. Plant Mol. Biol. 86, 139-156. doi: 10.1007/s11103-0140218-5

Tan, B. C., Joseph, L. M., Deng, W. T., Liu, L. J., Li, Q. B., Cline, K., et al. (2003). Molecular characterization of the Arabidopsis 9-cis epoxycarotenoid dioxygenase gene family. Plant J. 35, 44-56. doi: 10.1046/j.1365-313X.2003. 01786.x

Tan, B. C., Schwartz, S. H., Zeevaart, J. A. D., and McCarty, D. R. (1997). Genetic control of abscisic acid biosynthesis in maize. Proc. Natl. Acad. Sci. U.S.A. 94, 12235-12240. doi: 10.1073/pnas.94.22.12235

Tasseva, G., Richard, L., and Zachowski, A. (2004). Regulation of phosphatidylcholine biosynthesis under salt stress involves choline kinases in Arabidopsis thaliana. FEBS Lett. 566, 115-120. doi: 10.1016/j.febslet.2004. 04.015
Trapnell, C., Williams, B. A., Pertea, G., Mortazavi, A., Kwan, G., van Baren, M. J., et al. (2010). Transcript assembly and quantification by RNA-Seq reveals unannotated transcripts and isoform switching during cell differentiation. Nat. Biotechnol. 28, 511-515. doi: 10.1038/nbt.1621

Tuteja, N. (2007). Abscisic Acid and abiotic stress signaling. Plant Signal. Behav. 2, 135-138. doi: 10.4161/psb.2.3.4156

Valliyodan, B., and Nguyen, H. T. (2006). Understanding regulatory networks and engineering for enhanced drought tolerance in plants. Curr. Opin. Plant Biol. 9, 189-195. doi: 10.1016/j.pbi.2006.01.019

Vriet, C., Russinova, E., and Reuzeau, C. (2012). Boosting crop yields with plant steroids. Plant Cell 24, 842-857. doi: 10.1105/tpc.111.09 4912

Wang, H. Y., Wang, H. L., Shao, H. B., and Tang, X. L. (2016). Recent advances in utilizing transcription factors to improve plant abiotic stress tolerance by transgenic technology. Front. Plant Sci. 7:67. doi: 10.3389/fpls.2016. 00067

Wang, M. L., Wang, Y., Wu, H. Q., Xu, J., Li, T. T., Hegebarth, D., et al. (2016). Three TaFAR genes function in the biosynthesis of primary alcohols and the response to abiotic stresses in Triticum aestivum. Sci. Rep. 6:25008. doi: 10.1038/ srep25008

Wang, W. J., Liu, X. W., Gai, X. S., Ren, J. J., Liu, X. F., Cai, Y. L., et al. (2015). Cucumis sativus L. WAX2 Plays a pivotal role in wax biosynthesis, influencing pollen fertility and plant biotic and abiotic stress responses. Plant Cell Physiol. 56, 1339-1354. doi: 10.1093/pcp/pcv052

Xiong, L. M., Ishitani, M., Lee, H., and Zhu, J. K. (2001). The Arabidopsis LOS5/ABA3 locus encodes a molybdenum cofactor sulfurase and modulates cold stress- and osmotic stress-responsive gene expression. Plant Cell 13, 2063-2083. doi: 10.1105/tpc.13.9.2063

Young, M. D., Wakefield, M. J., Smyth, G. K., and Oshlack, A. (2010). Gene ontology analysis for RNA-seq: accounting for selection bias. Genome Biol. 11:R14. doi: 10.1186/gb-2010-11-2-r14

Zhang, M. L., Kong, X. P., Xu, X. B., Li, C. L., Tian, H. Y., and Ding, Z. J. (2015). Comparative transcriptome profiling of the maize primary, crown and seminal root in response to salinity stress. PLOS ONE 10:e0121222. doi: 10.1371/journal. pone.0121222

Zhang, X., Wang, L., Meng, H., Wen, H. T., Fan, Y. L., and Zhao, J. (2011). Maize ABP9 enhances tolerance to multiple stresses in transgenic Arabidopsis by modulating ABA signaling and cellular levels of reactive oxygen species. Plant Mol. Biol. 75, 365-378. doi: 10.1007/s11103-011-9732-x

Conflict of Interest Statement: The authors declare that the research was conducted in the absence of any commercial or financial relationships that could be construed as a potential conflict of interest.

Copyright (C) 2017 Li, Cao, Fang, Xu, Yin, Zhang, Lin, Wang, Chen, Xu and Yang. This is an open-access article distributed under the terms of the Creative Commons Attribution License (CC BY). The use, distribution or reproduction in other forums is permitted, provided the original author(s) or licensor are credited and that the original publication in this journal is cited, in accordance with accepted academic practice. No use, distribution or reproduction is permitted which does not comply with these terms. 